\title{
Methodological Challenges in Eye-Tracking based Usability Testing of 3-Dimensional Software - Presented via Experiences of Usability Tests of Four 3D Applications
}

\author{
Mária Babicsné-Horváth ${ }^{1 *}$, Károly Hercegfi ${ }^{1}$ \\ 1 Department of Ergonomics and Psychology, Faculty of Economic and Social Sciences, Budapest University of Technology and \\ Economics, H-1521 Budapest, P.O.B. 91, Hungary \\ * Corresponding author, e-mail: babicsne.horvath.maria@gtk.bme.hu
}

Received: 07 July 2020, Accepted: 05 January 2021, Published online: 03 January 2022

\begin{abstract}
Eye-tracking based usability testing and User Experience (UX) research are widespread in the development processes of various types of software; however, there exist specific difficulties during usability tests of three-dimensional (3D) software. Analysing the screen records with gaze plots, heatmaps of fixations, and statistics of Areas of Interests (AOI), methodological problems occur when the participant wants to rotate, zoom, or move the 3D space. The data gained regarded the menu bar is mainly interpretable; however, the data regarded the 3D environment is hardly so, or not at all. Our research tested four software applications with the aforementioned problem in mind: ViveLab and Jack Digital Human Modelling (DHM) and ArchiCAD and CATIA Computer Aided Design (CAD) software. Our original goal was twofold. Firstly, with these usability tests, we aimed to identify issues in the software. Secondly, we tested the utility of a new methodology which was included in the tests. This paper summarizes the results on the methodology based on individual experiments with different software applications. One of the main ideas behind the methodology adopted is to tell the participants (during certain subtasks of the tests) not to move the 3D space while they perform the given tasks at a certain point in the usability test. During the experiments, we applied a Tobii eye-tracking device, and after the task completion, each participant was interviewed. Based on these experiences, the methodology appears to be both useful and applicable, and its visualisation techniques for one or more participants are interpretable.
\end{abstract}

\section{Keywords}

usability testing, eye-tracking, Vivelab, Jack, ArchiCAD, CATIA, 3D environment

\section{Introduction}

Eye-tracking methodology is a standard tool among researchers. There exist different types of eye-tracking devices: eye-trackers integrated into monitors, portable eye-trackers, or mobile eye-tracking glasses. Different eye-trackers can be used in different scientific areas. One of the most common areas is Human-Computer Interaction (HCI). In this field, eye-tracking can be used, for instance, in software development or web application testing (Kim et al., 2018). Many different visualisation techniques can be used during post-processing eye-tracking data, such as heatmap (Tula et al., 2016), or gaze plot (Räihä et al., 2005). However, for an aggregated visualisation, the screens of the participants must look the same. In a usability test of a website or a traditional software application, the separated tasks allow researchers to select a specific part of each participant's timeline, so in the two-dimensional (2D) environment, visualisation techniques are interpretable (Jowers et al., 2013). However, where a more complicated software with a three-dimensional (3D) environment, like a Computer Aided Design (CAD) software, is being tested, participants can rotate, zoom in/out, or translate the $3 \mathrm{D}$ environment during task completion. Therefore, most of the visualisation techniques (gaze plots and heatmaps) and the statistics of Areas of Interests (AOI) (Józsa and Hámornik, 2011) are not interpretable in case of the inner $3 \mathrm{D}$ workspace (the huge inner area of the screen). The eye-tracking data on the menu bar is interpretable; however, it can hardly be understood in the 3D environment.

Recent findings on a video game conducted by the researchers of the University of Aveiro, Portugal highlight the same problem (Almeida et al., 2016). In one 
of our previous studies, we tested a Digital Human Modelling (DHM) software application, and we encountered the same problem: the data of the aggregated heatmaps and gaze plots were not interpretable (Babicsné Horváth et al., 2019).

In our present research, we made a methodological change which could potentially be a solution to this problem. We tested the interface of four pieces of software from a usability perspective: ViveLab and Jack DHM software are used for ergonomic simulation and risk assessment; ArchiCAD and CATIA are CAD software for architectural and mechanical engineering and design.

Performing the tests, our goal was twofold. Firstly, with these usability tests, we aimed to identify issues in the software. Secondly, we tested the utility of a new methodology which was included in the tests. Our research team have some usability issues concerning the two DHM software applications, which the authors of this paper have already published about (Babicsné-Horváth and Hercegfi, 2019). This paper focuses on the results of the methodology summarising the experiences of the individual experiments testing the distinct software.

\section{Methods and tools}

Several techniques were used to identify usability problems of the four pieces of software. Before performing the usability tests, the participants were asked a few basic questions to relieve tension. During the experiment, we applied eye-tracking methodology, and at the end, the experimenter interviewed each participant.

\subsection{Eye-tracking as a usability testing technique}

In HCI, usability testing was used first around 1980 (Dumas and Redish, 1999). The goal of a usability test is to evaluate a service or product. To reach this goal, researchers have to make a series of test sessions with representative users. The commonly used elements in usability tests are, for instance, observation, video and audio recording, taking photographs, and taking notes while participants try to perform the given tasks; furthermore, after the task completion, interviews are usually done. The aim is to identify usability issues, collect qualitative and quantitative data related to them, and determine the product/service satisfaction of the participants. (Riihiaho, 2017)

Eye-tracking methodology in HCI is a widely used tool among researchers for measuring usability and user experience (UX) (Ghaoui, 2006). Various types of research have been conducted in the field of web design (Herendy, 2009; Herendy, 2018; Józsa, 2010; Romano Bergstrom et al., 2013), and other HCI fields (Józsa and Hámornik, 2011; Katona and Kovari, 2018; Michalski, 2018; Kvaszingerné Prantner, 2015; Tóth and Szabó, 2018; Ujbanyi et al., 2016). This methodology can give us additional information about the users' behaviour (Wang et al., 2019). Combining the mentioned usual usability test techniques (e.g., observation, video recording, event logging) with eye-tracking methods, researchers can gain more data, and the visualisation techniques can support the interpretation of the data in a relatively efficient way.

In our research, we used a monitor-based eye-tracking device (Tobii T120). The cameras built in the monitor not only can record the participants' movements, gestures, and facial expressions but can determine their gaze. The system also records the computer screen, and as a result, we can get a video with the eye movements and visualisations such as heatmaps and gaze plot diagrams of fixations and statistics of AOI.

\subsection{The four tested software}

\subsubsection{ViveLab}

ViveLab is a DHM software for ergonomic assessment: analysing the human motion and postures, generating risk assessment documents and deriving statistics. It was released in 2015, in Hungary. The software is cloud-based: the shared model spaces can be edited and used for analysis through a web browser-based thin client (ViveLab Ergo).

Its features include setting the human model, importing motion capture files, and manual creation of animations. The software has three implemented risk assessment methods - including the Rapid Upper Limb Assessment (RULA) used in our study-, two implemented standards (ISO 11226, EN 1005-4), and two other analysis techniques (reachability zone, spaghetti diagram).

Our study tested the latest version of ViveLab as of 2019.

\subsubsection{Jack}

Jack software was developed at the University of Pennsylvania in the 1980s for the simulation of military actions and maintenance work. Nowadays, Jack can be considered as an industry standard software for ergonomics (Blanchonette, 2009). Today it is a part of the portfolio of Siemens for digital manufacturing. The earlier Siemens Product Lifecycle Management (PLM) software line has recently rebranded as Tecnomatix, and the earlier features of Jack appears as three modules: Tecnomatix Jack, Tecnomatix Motion Capture Toolkit, and Tecnomatix Process Simulate Human.

In our study, we tested the 8.0.1 version of Jack. 
Jack's virtual environment allows us to import CAD models. Information such as the distance of two points or access zones can be displayed. Motion capture allows us to incorporate the movement of a real person into the human model. Simulations can be done, too. It can also generate reports based on exact results. Analysis as reachability zones, RULA, and other tools are also available in Jack.

\subsubsection{ArchiCAD}

ArchiCAD (Fischer and Fischer, 2012) is a tool for architects for designing buildings. It is developed by Graphisoft, allowing architects to work with Building Information Modelling (BIM) (Jung and Joo, 2011; Volk et al., 2014). It has a series of advantages, like dealing with a complex information model of virtual buildings, working in 3D environment, real-time rendering, automatically generated documents, and data sharing for teamwork.

The present paper summarises the methodological results of distinct usability studies. The usability test of the ArchiCAD was our first experience with the mentioned 3D methodological problem in eye-tracking; consequently, an old version was tested: ArchiCAD 16.

\subsubsection{CATIA}

CATIA is a software application developed by the French company, Dassault Systèmes. The development of the application first started in 1977. Initially, it was a software used to design Dassault Mirage 2000 aircraft, but later it was adapted to other areas (such as shipbuilding and car production). CATIA supports various stages of product development, including conceptualisation, design (CAD), Computer Aided Engineering (CAE) and Computer Aided Manufacturing (CAM). In this research, its CAD affordances were tested. CATIA offers a solution to shape design, styling, surfacing workflow and visualisation to create, modify, and validate complex shapes.

Our experiments performed in 2020 tested the CATIA Version 5.

\section{Participants and protocols of the usability tests}

During the recruitment of participants, we paid attention to the user profile. The previous experience was the most crucial aspect. Regarding ViveLab and Jack, we searched for participants who are familiar with the field of anthropometry and ergonomic risk assessments. Regarding ArchiCAD, it was important to be an architect or architect student knowing the software, and regarding CATIA, it was essential to be familiar with 3D modelling as a mechanical engineer or a designer.
The structures of the usability tests were similar. First, the calibration of the Tobii T120 eye-tracker was made. After that, the participants had to complete the given tasks.

\subsection{Protocol of the usability tests of ArchiCAD}

The usability test was focusing on the new features of ArchiCAD 16, and the differences compared to the previous versions. The seven participants of the usability test were architect students from the local university, because they were easy to access, and they had previous experience of using ArchiCAD. The task completion time was around 45-60 minutes.

The tasks for the participants were the following:

- Free moving. The participants were asked to move a box to different points of the 3D environment.

- Choose a sub-item. In this part, editing with the new morph tool was examined.

- Convert to shape. In this case, the participants were asked to make an arched object from a flat shape.

- Fit to surface. The participants were asked to make a wall between two walls, then extend the previously created wall.

- Door modification. This task was made in ArchiCAD 15 because of language issues.

We tried to avoid the change of views by giving the task instructions step by step, via a view from which the task can also be performed. Moreover, during the main phases, we asked the participants not to modify the view. Searching among the menus was the main point of the test. Focusing on this short period of the test, we can evaluate heatmaps and gaze plots by the help of the eye-tracking methodology.

\subsection{Protocol of the usability tests of ViveLab and Jack}

Regarding the task completion of ViveLab and Jack software, in each session, the sequence of the two software was randomly chosen (however, we paid attention to the equal number of the users testing with each order). Eventually, four participants started the tasks with ViveLab, and four started with Jack. Consequently, the learnability effect was not always the same.

Before the usability tests, a few adjustments were made. We created eight separated "virtual labs" (shared model spaces) in ViveLab for the eight participants. Firstly, a CAD model of a roller conveyor was added. Secondly, a viewpoint was defined (with the same view) in every lab. We also created a rectangular solid representing the roller conveyor in Jack, which was necessary due to methodological considerations. 
We shortened the task list after a pilot test. The task completion of the pilot took one and a half hour, which was tiring for the pilot participant. After correcting the protocol based on the experiences of the pilot, the average task completion time proved to be 45 minutes.

The tasks were the following:

- Open ViveLab/Jack.

- Try how you can move, rotate the 3D space, and zoom.

- Create a human model and set the given parameters.

- Find the viewpoint named "Viewpoint 1" and insert the camera (we asked the participants not to move the camera for the next two tasks).

- Create a $10 \mathrm{~cm} \times 10 \mathrm{~cm} \times 10 \mathrm{~cm}$ cube (illustrating the workpiece).

- Adjust the colour/transparency of the cube. (After this task, the participants were allowed to move the camera.)

- Adjust the position of the human and the cube without moving the roller bar.

- Turn on the RULA Risk Assessment Panel. The task is over when they read aloud what point the posture has got.

- Make an animation in which the human lifts the cube, raises it closer to the eye (as visual inspection).

- Play the animation from start to finish.

- Turn on the RULA Risk Assessment Panel and check the score of the body posture when the human lifts the cube.

Searching in the menus was the main point of the test. We tried to avoid the change of views by giving the tasks step by step, in a view from which the task can be performed. Also, we asked the participants not to modify the view during two tasks. Focusing on this short period of the test, we can evaluate heatmaps and gaze plots by the help of the eye-tracking methodology.

\subsection{Protocol of the usability tests of CATIA}

The usability tests of CATIA were focusing on previously suspected problems. This experiment remained in an early stage (performing a pilot test) because of the Corona Virus Disease (COVID-19) situation of 2020; however, the methodological results regarding the topic of this paper can already be analysed.

The tasks were the following:

- Try how you can move, rotate the 3D space, and zoom.
- Find the "Isometric view", and the "Fit all in" icons. (We asked the participant not to move the camera for the next two tasks.)

- Make an extraction (with the given parameters) (after this task, the participant could move the camera).

- Cut a rectangle (with the given parameters).

- Use the Hole command (with the given parameters).

- Make fillets (with the given parameters).

The task completion time was one hour.

\section{Results}

As the results of the usability tests, we have identified many usability problems regarding the four pieces of software. Two types of data can be gained: qualitative and quantitative. The main qualitative data came from eye-tracking visualisation techniques (heatmaps, gaze plots). The quantitative data came from the task and subtask completion times and the success rate. In this paper, we are focusing on methodological problems and successes. The results regarding the usability of the different software tests were and shall be written about in another article.

During specific phases of all the usability tests, participants were asked not to move the 3D space for a while. During those phases, the camera views of the participants were the same or very similar to the others'. Due to this methodological speciality, more gained data could be interpreted by the help of the visualisation techniques of the eye-tracking. However, unexpected problems occurred, too.

\subsection{Methodological problems of research to solve}

In this section, the difficulties regarding the methodology are discussed. In many cases, we found small differences between two problem solutions, which resulted that the aggregated heatmaps are not or just partially interpretable.

\subsubsection{Viewpoint setting}

Preparing the usability tests, we defined a viewpoint in each software. In Jack, it was easy, because the coordinates of the viewpoint could be defined and when participants opened the software, they found the same viewpoint. In ViveLab, it was harder, because we created a new "virtual lab" for every participant and the coordinates of the viewpoint cannot be defined numerically. It could have caused a problem, however, fortunately, similar viewpoints were created. In CATIA, there was no viewpoint created; however, the isometric view and using the "Fit all in" command would give the same view. Unfortunately, the pilot 
test showed that it could be different (Fig. 1). Setting up the viewpoint is a crucial part of this methodology.

\subsubsection{Popup windows}

During the task completion, the popup windows could appear in different places for the different participants. In many software applications, the positioning of the popup window can be defined (e.g. by setting up the environment in advance). However, participants still can drag the windows. In this case, the views of the participants are not the same, meaning that the aggregated heatmaps will be unusable.

Fig. 2 shows an example from the test of CATIA: The pilot participant tried everything and could not solve the task, so he dragged the popup window.

Another example, where the popup window appeared in different places for two participants. Fig. 3 shows that problem occurring during the test of Jack.

\subsubsection{Context menus}

During performing the usability tests, many context menus were used. Firstly, the place of the context menu can depend on where the user clicks. If it is a line or a large
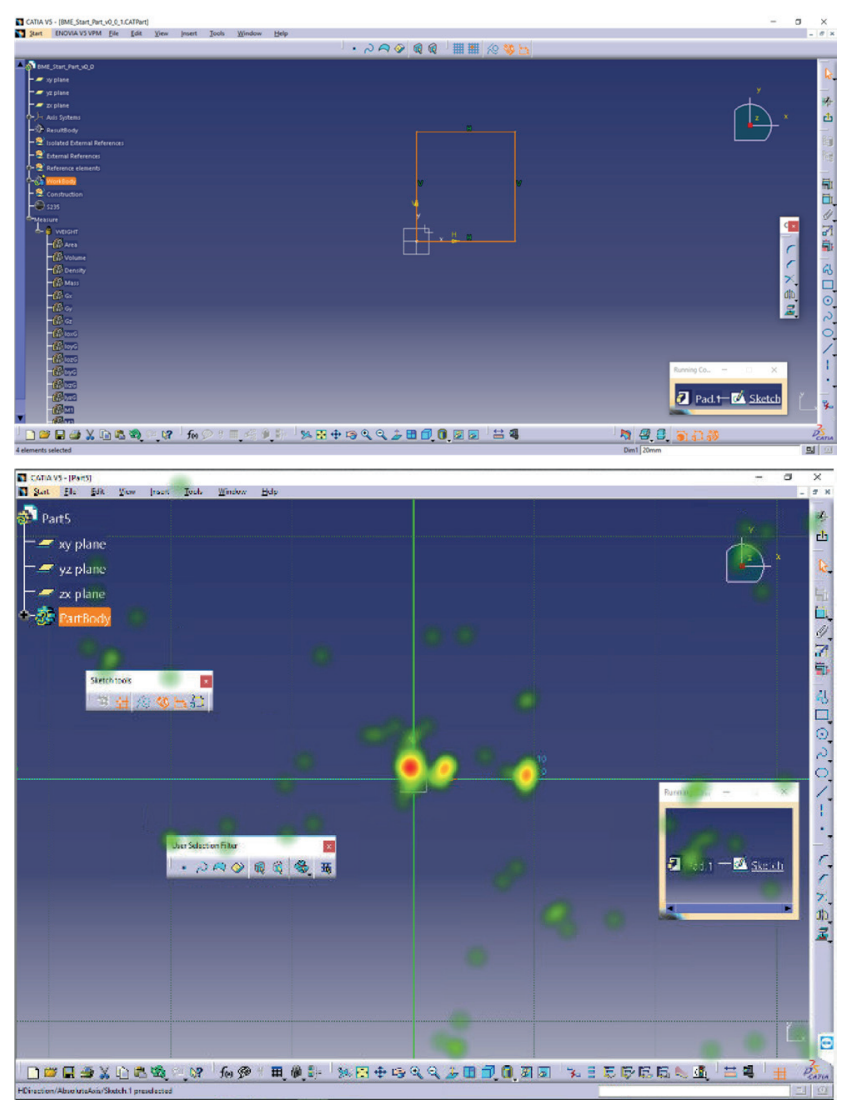

Fig. 1 Two different viewpoints in Catia, preparing the test (upper screenshot) and completing the test (lower screenshot with heatmap of fixations)


Fig. 2 Dragging of popup window in CATIA causes difficulties in interpretation of heatmaps
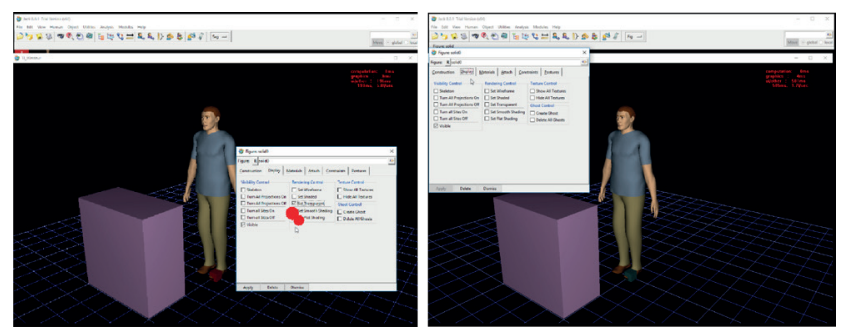

Fig. 3 Appearing a popup window in different places in Jack causes difficulties in interpretation of gaze plots

object, they can click on it in different places. Secondly, context menus can appear in different places whether specific panels of the user interface (UI) are on or off. Even so, if more than one participant has seen the same picture, eye-tracking data can be aggregated. For instance, Fig. 4 shows that in ViveLab, the appearance of the panel on the right side did prove to be important.

In ArchiCAD, participants could click on different places of the object. Consequently, the context menu appeared in different places, as can be seen in Fig. 5. In this case, the positions of the context menus were only slightly different, therefore, the aggregation can be analysed only carefully.

\subsubsection{Drop-down menus}

Especially in Jack, many drop-down menus can be used. The users can reach them from the upper menu bar. More than one task can be solved by the mentioned UI element.

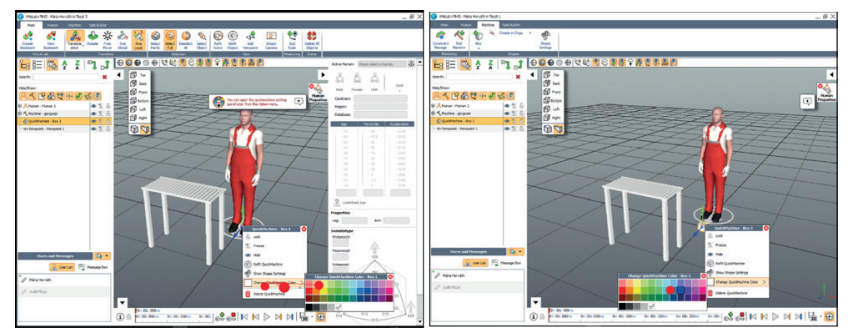

Fig. 4 Context menu on different places in ViveLab in function of whether the right panel is on (left gaze plot) or off (right gaze plot). The different positions of the context menu causes difficulties in interpretation of gaze plots 




Fig. 5 Context menu appearing on different positions in ArchiCAD can cause difficulties in interpretation of heatmaps

Therefore, examining the static heatmap for a specific period, it looks as though sometimes the participants looked at the empty open space. Fig. 6 ostensibly shows that many fixations directed to weird (empty) areas.

\subsubsection{Reminder for the experimenter}

The experimenter must persistently pay attention to the possibility that the participants can forget the rule not to move, rotate, or zoom in/out the 3D space. Sometimes the participants need to be reminded.

\subsubsection{More than one solution}

Almost in all software, each task has many solutions. The variety of solutions can help the users to find their best and easiest way to complete the tasks. However, in our usability tests, from the viewpoint of the aggregated visualisations of the eye-tracking, it represents a difficulty. Where different solutions have been attempted, less data can be aggregated.

\subsection{Successes in methodology, interpretable heatmaps}

Although many problems have occurred, the methodology was successful in all usability tests. The problems can be



Fig. 6 Eye gazes on uninterpretable places in Jack because of the temporary usage of drop-down menus corrected with some more instruction or with corrections performed during post-processing of the results. Despite the previous issues, we were able to create aggregated and individual heatmaps, as they are presented below.

\subsubsection{Drop-down menus}

Despite of the mentioned problems caused by drop-down menus, Fig. 7 shows an example for an aggregated heatmap of Jack which can be evaluated. It shows where the participants looked at most of the time. We can conclude that the information in the drop-down menu was not clear for the participants, because they paid attention to all functions.

\subsubsection{Popup windows}

Examples can be found where the popup window occupies the entire screen, with the result that the participants do not move it. For instance, it occurs in ArchiCAD, in case of the door modification window (Fig. 8).

Fig. 9 shows another example in Jack, where the participants created animation.

\subsubsection{More than one solution}

The fact that there is more than one solution could be a problem, as previously mentioned. However, it does not block the creation and interpretation of heatmaps, it only reduces the number of heatmaps that can be merged. Therefore, if at least two participants choose the same solution, aggregated heatmaps can be created (Fig. 10).

\subsubsection{Beyond the menus and icons: Assessing the} interactions in the view of the model space

During the task completion, participants have to use the inner area of the screen, the graphic view of the model space,



Fig. 7 Creating a cube in Jack - aggregated heatmap for all participants 




Fig. 8 Door modification task in ArchiCAD. Heatmap of one participant

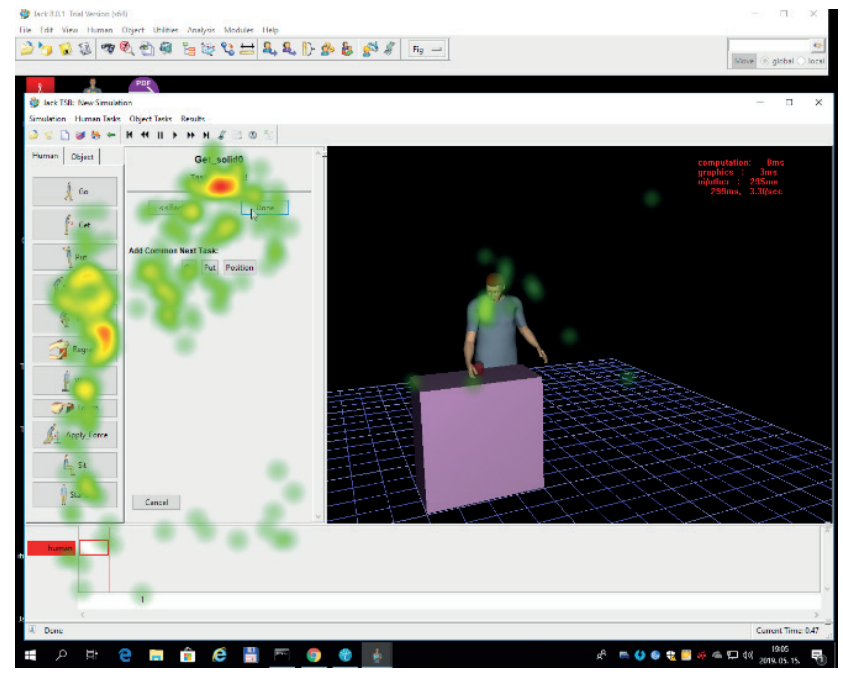

Fig. 9 Creating animation in Jack. Aggregated heatmap

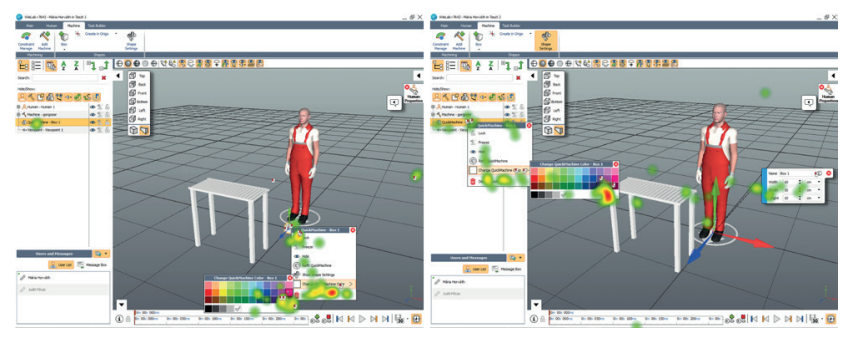

Fig. 10 Heatmaps of different solutions in ViveLab

where they have to interact with lines, objects, etc. Similar views allow us to aggregate the eye gaze data. Applying a static view (without moving, rotating, and zoom in/out the $3 \mathrm{D}$ space) helps us to analyse a wider time period in its complexity. Fig. 11 shows an example from the test of CATIA when the participant searched for the clickable point of the right line of the cube to make the required chamfer.

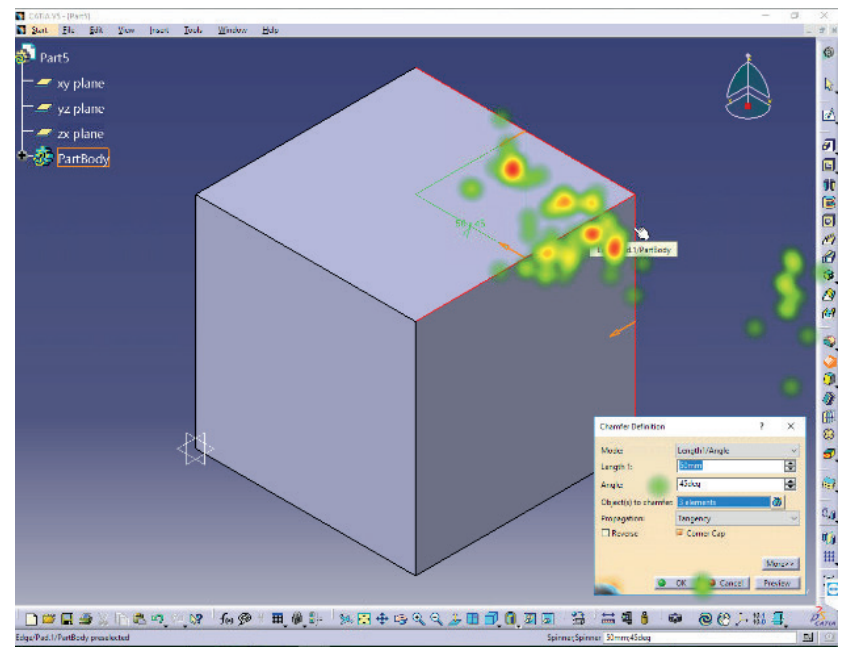

Fig. 11 Searching for a line to chamfer in CATIA - heatmap of one participant

\section{Conclusion and discussion}

In conclusion, the applied usability testing method has been proved useful. Based on the eye-tracking data, suggestions for software development can be made. Furthermore, based on our experiences published here, development suggestions for the development of the methodology can also be made. Regarding the software, development suggestions are not concluded in this paper.

The problems of the eye-tracking methodology in 3D environments are solvable, but with compromises. During the analysis of tests applying eye-tracking in 3D environments, the hardest problem is that the most sophisticated, aggregated visualisations, such as aggregated heatmaps, can only be used in restricted situations, so they are not always suitable for modelling natural user behaviour. While zooming in and out and moving and rotating the space and rotation make the task completion of the user easier, they make the analysis more challenging. Despite when the participants were asked not to move and rotate the $3 \mathrm{D}$ space and not to zoom, they could open context menus on different places, therefore, in some cases, the aggregated heatmaps and gaze plots would not be interpretable.

The tested four pieces of software are massively $3 \mathrm{D}$-based, which is one of the reasons why we chose them. The different interfaces of the four software showed the many different difficulties which can occur applying eye-tracking in 3D software.

With this research, we intend to give suggestions for other researchers on how to complete similar eye-tracking based usability tests in cases of 3D software with satisfactory results. Our suggestions, which conclude the original ideas and the findings, are the following: 
- Give precise tasks.

- Avoid possible differences. Give instructions for most cases (for instance, open or close specific panels).

- Watch out for the popup windows. We cannot give an overall solution for the problem of the popup windows in different positions, but the experimenter can ask (for some task or subtasks) the participants not to move these windows for a better result.

- Freeze the 3D environment or ask the participant not to move it at least for some tasks. A good definition of a common viewpoint is crucial.

- Beyond the 3D space, the positions of the models are also important. Give exact instructions on whether they can move the model or not, and where should they create sketches.

- Do pilot tests. It is obviously a good idea to do a pilot test; however, in this case, it is crucial.

- Check the monitor ratio. Is there enough place to solve the tasks?

\section{References}

Almeida, S., Mealha, Ó., Veloso, A. (2016) "Video game scenery analysis with eye tracking", Entertainment Computing, 14, pp. 1-13. https://doi.org/10.1016/j.entcom.2015.12.001

Babicsné-Horváth, M., Hercegfi, K. (2019) "Early Results of a Usability Evaluation of Two Digital Human Model-based Ergonomic Software Applying Eye-Tracking Methodology Comparison of the usability of ViveLab and Jack software", In: 10th IEEE International Conference on Cognitive Infocommunications (CogInfoCom), Naples, Italy, pp. 205-210. https://doi.org/10.1109/CogInfoCom47531.2019.9089993

Babicsné Horváth, M., Hercegfi, K., Fergencs, T. (2019) "Comparison of Digital Human Model-Based Ergonomic Software Using EyeTracking Methodology - Presenting Pilot Usability Tests", In: Duffy, V. (ed.) Digital Human Modeling and Applications in Health, Safety, Ergonnomics and Risk Management. Human Body and Motion. HCII 2019. Lecture Notes in Computer Science, Springer, Cham, Switzerland, pp. 22-32.

https://doi.org/10.1007/978-3-030-22216-1_2

Blanchonette, P. (2009) "Jack Human Modelling Tool: A Review", Australian Government, Department of Defence, Defence Science and Technology Organisation, Victoria, Australia, Rep. DSTO-TR-2364, 2009.

Dumas, J. S., Redish, J. C. (1999) "A Practical Guide to Usability Testing", Intellect, Exeter, UK.

Fischer, F., Fischer, K. (2012) "ARCHICAD: Einführung und Nachschlagewerk" (ARCHICAD: Introduction and reference work), Springer Vieweg+Teubner Verlag, Wiesbaden, Deutschland. (in German)

https://oi.org/10.1007/978-3-8348-2220-8
- Leave more time to the post-processing and evaluation.

Summarising the research, we can conclude that the methodology is useful and can be applied in other similar usability tests. The visualisation techniques for individual participants are interpretable. However, the aggregated visualisations are interpretable only in special cases.

\section{Acknowledgement}

The authors thank Tímea Varga for her earlier research activity regarding ArchiCAD, Réka Miklós, Rita Borbála Ferenczy, and Trang Ha Ngo for preparation of the task list for the test of CATIA, and the participants for their valuable contributions.

This research was supported by the New National Excellence Programme (ÚNKP) of the National Research, Development and Innovation Office of the Hungarian Government.

Ghaoui, C. (2006) "Encyclopedia of Human Computer Interaction", IGI Global, Pennsylvania, USA.. https://doi.org/10.4018/978-1-59140-562-7

Herendy, C. (2009) "How to Research People's First Impressions of Websites? Eye-Tracking as a Usability Inspection Method and Online Focus Group Research", In: Godart, C., Gronau, N., Sharma, S., Canals, G. (eds.) Software Services for e-Business and e-Society, I3E 2009, IFIP Advances in Information and Communication Technology, Springer, Heidelberg, Berlin, Germany, pp. 287-300. https://doi.org/10.1007/978-3-642-04280-5_23

Herendy, C. (2018) "How to Learn About Users and Understand Their Needs? User Experience, Mental Models and Research at Public Administration Websites", Socialiniai tyrimai: Social Research, 41(1), pp. 5-17. https://doi.org/10.21277/st.v41i1.241

Jowers, I., Prats, M., McKay, A., Garner, S. (2013) "Evaluating an eye tracking interface for a two-dimensional sketch editor", ComputerAided Design, 45(5), pp. 923-936. https://doi.org/10.1016/j.cad.2013.01.006

Józsa, E., Hámornik, B. P. (2011) "Find the Difference! Eye Tracking Study on Information Seeking Behavior Using an Online Game", Journal of Eye Tracking, Visual Cognition and Emotion, 2(1), pp. 27-35.

Józsa, E. (2010) "A potential application of pupillometry in web-usability research", Periodica Polytechnica Social and Management Sciences, 18(2), pp. 109-115. https://doi.org/10.3311/pp.so.2010-2.06

Jung, Y., Joo, M. (2011) "Building information modelling (BIM) framework for practical implementation", Automation in Construction, 20(2), pp. 126-133.

https://doi.org/10.1016/j.autcon.2010.09.010 
Katona, J., Kovari, A. (2018) "The Evaluation of BCI and PEBL-based Attention Tests", Acta Polytechnica Hungarica, 15(3), pp. 225-249. https://doi.org/10.12700/aph.15.3.2018.3.13

Kim, E., Tang, L. R., Meusel, C., Gupta, M. (2018) "Optimization of menu-labeling formats to drive healthy dining: An eye tracking study", International Journal of Hospitality Management, 70, pp. 37-48. https://doi.org/10.1016/j.ijhm.2017.10.020

Kvaszingerné Prantner, C. (2015) "The evaluation of the results of an eye tracking based usability tests of the so called Instructor's Portal framework (http://tanitlap.ektf.hu/csernaiz)", In: 6th IEEE International Conference on Cognitive Infocommunications (CogInfoCom), Gyor, Hungary, pp. 459-465. https://doi.org/10.1109/CogInfoCom.2015.7390637

Michalski, R. (2018) "Information presentation compatibility in a simple digital control panel design: eye-tracking study", International Journal of Occupational Safety and Ergonomics, 24(3), pp. 395-405. https://doi.org/10.1080/10803548.2017.1317469

Räihä, K. J., Aula, A., Majaranta, P., Rantala, H., Koivunen, K. (2005) "Static Visualization of Temporal Eye-Tracking Data", In: Costabile, M. F., Paternò, F. (eds.) Human-Computer Interaction INTERACT 2005, Lecture Notes in Computer Science, Springer, Heidelberg, Berlin, Germany, pp. 946-949. https://doi.org/10.1007/11555261_76

Riihiaho, S. (2017) "Usability Testing", In: Norman, K., Kirakowski, J. (eds.) The Wiley Handbook of Human Computer Interaction, Wiley Blackwell, Hoboken, NJ, USA, pp. 255-275. https://doi.org/10.1002/9781118976005.ch14

Romano Bergstrom, J. C., Olmsted-Hawala, E. L., Jans, M. E. (2013) "Age-Related Differences in Eye Tracking and Usability Performance: Website Usability for Older Adults", International Journal of Human-Computer Interaction, 29(8), pp. 541-548. https://doi.org/10.1080/10447318.2012.728493
Tóth, Á., Szabó, B. (2018) "A Pilot Research on Sport application's Usability and Feedback Mechanics", In: 9th IEEE International Conference on Cognitive Infocommunications, (CogInfoCom), Budapest, Hungary, pp. 75-80. https://doi.org/10.1109/CogInfoCom.2018.8639870

Tula, A. D., Kurauchi, A., Coutinho, F., Morimoto, C. (2016) "Heatmap Explorer: an interactive gaze data visualization tool for the evaluation of computer interfaces", In: IHC '16: 15th Brazilian Symposium on Human Factors in Computer Systems, São Paulo, Brazil, Article number: 24. https://doi.org/10.1145/3033701.3033725

Ujbanyi, T., Katona, J., Sziladi, G., Kovari, A. (2016) "Eye-tracking analysis of computer networks exam question besides different skilled groups", In: 7th IEEE International Conference on Cognitive Infocommunications (CogInfoCom), Wroclaw, Poland, pp. 277-282.

https://doi.org/10.1109/CogInfoCom.2016.7804561

ViveLab Ergo "ViveLab Ergonomic Software", [online] Availabe at: http://vivelab.cloud/ [Accessed: 10 December 2019]

Volk, R., Stengel, J., Schultmann, F. (2014) "Building Information Modeling (BIM) for existing buildings - Literature review and future needs", Automation in Construction, 38, pp. 109-127. https://doi.org/10.1016/j.autcon.2013.10.023

Wang, J., Antonenko, P., Celepkolu, M., Jimenez, Y., Fieldman, E., Fieldman, A. (2019) "Exploring Relationships Between Eye Tracking and Traditional Usability Testing Data", International Journal of Human-Computer Interaction, 35(6), pp. 483-494. https://doi.org/10.1080/10447318.2018.1464776 